\section{Caracterización clínica e imagenológica de una serie de pacientes con disección de arterias cervicales e intracraneales}

\author{
ANDRÉS GALLARDO V., ${ }^{1,3}$, RENÉ NÚÑEZ F. ${ }^{2}$, OSVALDO TRUJILLO ${ }^{1,4}$, \\ BENJAMÍN RODRÍGUEZ ${ }^{\mathrm{a}}$, FELIPE JURADO ${ }^{1,4}$, \\ HARUMI ASAHI ${ }^{1}$, CLAUDIA LAZCANO ${ }^{1}$, ÁLVARO ROMERO ${ }^{1}$, \\ MARÍA JESÚS SOTO ${ }^{1}$, ROQUE VILLAGRA ${ }^{1,3}$
}

\section{Cervicocranial arterial dissection. Experience in 57 patients}

Background: Cervicocranial arterial dissection (CIAD) is an important cause of stroke. Aim: To describe the clinical and imaging characteristics of patients with CIAD. Material and Methods: An anonymous registry was made including all patients admitted to a private hospital with a diagnosis of CIAD. Patients were subdivided as having an anterior or posterior circulation dissection ( $A C D$ or $P C D$, respectively). Results: Fifty-seven patients aged $40 \pm$ 8 years (60\% women) were included in the study, 39 with PCD and 18 with $A C D$. Cervical pain was the most common symptom. CIAD was diagnosed with no clinical or imaging signs of stroke in $49 \%$ of patients. Fifty one percent of patients had focal neurological deficits and 72\% had a NIH stroke score below five. No significant differences between patients with ACD or PCD were found. Fifty patients received antiplatelet therapy (simple or dual), seven patients were anticoagulated and 13 were subjected to stenting due to progression of stenosis with hemodynamic involvement or bilateral dissection with scarce collaterals. The lesion was located in V3 segment in 27 patients and cervical segment of the internal carotid in 16 cases. A favorable Modified Rankin Scale (0-2) was achieved in $85.9 \%$, with a trend towards achieving better functional prognosis in PCD. Conclusions: Due to the greater availability of non-invasive imaging methods, 50\% of these patients with CIAD did not have a stroke. Thus, an earlier and more timely management is feasible.

(Rev Med Chile 2021; 149: 1157-1163)

Key words: Carotid Artery, Internal, Dissection; Stroke; Vertebral Artery Dissection.

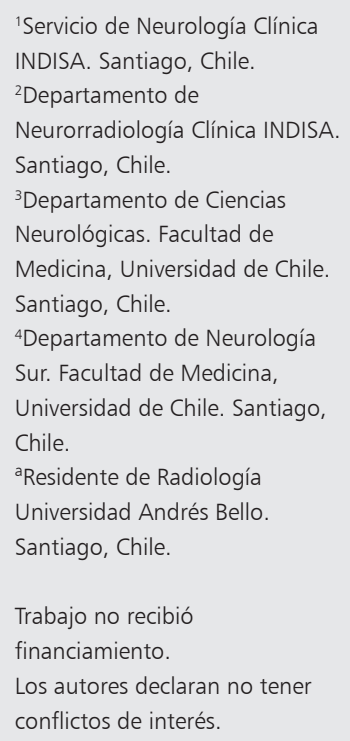

Recibido el 23 de octubre de 2020, aceptado el 7 de mayo de 2021.

Correspondencia a:

Dr. Andrés Gallardo Vera Departamento de Ciencias Neurológicas, Facultad de Medicina, Universidad de Chile.

Santiago, Chile.

a.gallardo@uchile.cl

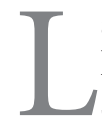

a disección arterial se desarrolla cuando la sangre ingresa a la pared de un vaso y separa las capas, lo que puede ser causado por un desgarro en la capa íntima o la ruptura del vasa vasorum en la túnica media ${ }^{1}$. La disección de arterias cervicales o intracraneales (DACI) es una etiología importante del accidente cerebro- vascular (ACV) en la juventud, ya que representa $25 \%$ de los eventos cerebrovasculares en pacientes menores de 45 años ${ }^{2}$. Aunque la DACI solía considerarse una entidad única, los hallazgos de estudios recientes sugieren que es un grupo de entidades patológicas con diversas características basadas en diferentes sitios de distribución ana- 
tómica, con distinto compromiso de la pared del vaso afectado y variadas formas de presentación clínica ${ }^{3}$.

$\mathrm{Al}$ reconocerse subgrupos dentro de esta entidad patológica, el manejo clínico en la práctica médica habitual puede diferir en consideración de las características del caso en particular, al no existir evidencia de una ventaja estadística de una terapia por sobre otra, cuando se considera al grupo de disección cervical de forma global ${ }^{4}$. Incluso a los estudios previamente realizados donde solo se consideraban como opciones terapéuticas la antiagregación y anticoagulación, ahora se adiciona la posibilidad de stenting en casos seleccionados ${ }^{5}$.

Si bien la recurrencia de disección y eventos cerebrovasculares es baja a mediano y largo pla$\mathrm{zo}^{6}$, el desenlace clínico en agudo puede diferir ampliamente en relación a las características del vaso disecado, del tratamiento con antiagregante plaquetario, anticoagulante o endovascular que se realice y de la evolución dentro de los primeros 10 días, al ser una entidad dinámica.

\section{Objetivo}

A) Describir las características clínicas e imagenológicas de ingreso.

B) Describir los tratamientos efectuados y la evolución clínica-imagenológica de los primeros 10 días.

C) Estratificar el outcome clínico de los pacientes en el control de seguimiento hasta los 3 meses, a través de la escala de Rankin modificada (ERm).

\section{Metodología}

Estudio de diseño descriptivo, cuya muestra corresponde a todos los pacientes mayores de 18 años cuyo motivo de hospitalización en Clínica INDISA fue una disección de arterias cervicales o intracraneales, con o sin ACV isquémico, en el periodo comprendido desde julio de 2017 hasta junio de 2020, excluyéndose a todos aquellos casos de duda diagnóstica inicial que posteriormente fueron descartados a través de una angiografía convencional.

Los pacientes que posterior a la realización de AngioTC o AngioRNM fueron diagnosticados con DACI, fueron ingresados a una base de da- tos clínicos, donde posteriormente se registran las variables a estudiar. Al finalizar el periodo de registro, el 30 de junio de 2020, se realizó el proceso de triple anonimización en conformidad a lo aprobado por el Comité de Ética del Servicio de Salud Metropolitano Oriente. Los pacientes con angiografía diagnóstica negativa, según la información ingresada en la base de datos, fueron eliminados de esta. Los datos fueron procesados por STATA versión 15.0. En variables cuantitativas se realizó prueba de Shapiro Wilk para normalidad, mientras que para las variables cualitativas se evaluó la asociación a través del test de chi2.

Se realizó un análisis global del total de disecciones y una división por subgrupo, agrupando a la disección de arterias de circulación anterior (DACA) y a las disecciones de arterias de circulación posterior (DACP).

Las variables a estudiar fueron:

a) Síntomas y signos de presentación, donde se registra la presencia de 1 o más de los siguientes: cervicalgia/cefalea, signo de Horner o déficit focal. Se registró la presencia de trauma en los últimos 30 días, definiéndose trauma menor como actividad física o Valsalva.

b) Características imagenológicas del vaso comprometido: detallándose el o los segmentos comprometidos, dividiéndose en territorio anterior o posterior, intracraneal o extracraneal y si lo que predomina es el hematoma subintimal o el hematoma subadventicial.

c) La presencia o no de evidencia de ACV isquémico al momento del ingreso, ya sea por clínica deficitaria o por lesión isquémica visible en secuencia DWI.

d) El o los tratamientos específicos realizados para resolver la patología, los cuales pueden ser antiagregación simple, antiagregación dual, anticoagulación o terapia endovascular.

e) Outcome clínico durante el seguimiento hasta los 3 meses, lo cual se define según el grado de funcionalidad física del paciente, basado en la ERm que va en un rango de 0 a 6 , donde 0 es asintomático, 5 es discapacidad grave y 6 es muerte. Se agrupa como Rankin favorable los pacientes con puntaje de 0 -2, considerándose como outcome desfavorable los pacientes con Rankin de 3-6.

f) Ocurrencia o recurrencia de isquemia desde las $72 \mathrm{~h}$ de hospitalización hasta los 3 meses de diagnosticada la disección. 


\section{Resultados}

Se lograron registrar en 3 años un total de 57 paciente con DACI, cuya edad promedio fue de 40 años, con una distribución mayor de mujeres que hombres de $59,6 \%$ vs $40,4 \%$ (Tabla 1 ). Treinta y nueve casos $(68,4 \%)$ correspondieron a disección de arterias que tradicionalmente irrigan la circulación posterior y $18(31,6 \%)$ arterias que irrigan la circulación anterior. A su vez, 13 pacientes (23\%) presentaron disección de vasos intracraneales, de ellos, 4 compromiso puramente intracraneal y 9 mixto.

El dolor estuvo presente en $87,7 \%$ del total de pacientes, sin diferencia significativa en relación a la localización de la disección (83,3\% en DACA, $89,7 \%$ en DACP) y el síndrome de Horner presen- te en $35 \%$ de los casos, siendo más prevalente en la disección carotidea (83,3\% en DACA, $12,8 \%$ en DACP). Veintinueve pacientes (50,9\%) ingresaron con un déficit focal o una lesión con restricción en difusión, sin diferencia significativa en relación a la localización de la disección (55,5\% en DACA, $48,7 \%$ en DACP). Del total de pacientes con DACI, $71,9 \%$ ingresó con NIHSS (National Institutes of Health Stroke Score) de 0-4, 8,8\% con NIHSS 5-9, $15,8 \%$ con NIHSS de $10-19,3,5 \%$ con NIHSS $\geq 20$ (Tabla 2).

En relación a los hallazgos imagenológicos de la disección, se encontró en $63,2 \%$ estenosis secundaria a hematoma mural, $24,6 \%$ oclusión, $10,5 \%$ dilatación aneurismal y en $14 \%$ las disecciones fueron bilaterales (Tabla 2).

La localización más frecuente en las DACP fue

Tabla 1. Características demográficas y factores de riesgo

\begin{tabular}{|lcccc|}
\hline & & Total & $\begin{array}{c}\text { Disección de circulación } \\
\text { anterior }\end{array}$ & $\begin{array}{c}\text { Disección de circulación } \\
\text { posterior }\end{array}$ \\
\hline $\mathrm{n}$ de pacientes & & 57 & 18 & 39 \\
Edad & $(\mathrm{DE})$ & $40(8,2)$ & $43(8,3)$ & $38(8,1)$ \\
\hline Mujeres & $\mathrm{n}(\%)$ & $34(59,6)$ & $6(33,3)$ & $28(71,8)$ \\
Trauma menor & $\mathrm{n}(\%)$ & $14(24,5)$ & $4(22,2)$ & $10(25,6)$ \\
Trauma mayor & $\mathrm{n}(\%)$ & $1(1,8)$ & $1(5,5)$ & 0 \\
\hline
\end{tabular}

Tabla 2. Características clínicas e imagenológicas

\begin{tabular}{|c|c|c|c|c|c|}
\hline & & Total & $\begin{array}{l}\text { Disección de } \\
\text { circulación anterior }\end{array}$ & $\begin{array}{l}\text { Disección de } \\
\text { circulación posterior }\end{array}$ & Valor $\mathbf{p}$ \\
\hline $\mathrm{n}$ de pacientes & & 57 & 18 & 39 & \\
\hline Dolor & n (\%) & $50(87,7)$ & $15(83,3)$ & $35(89,7)$ & 0,387 \\
\hline Horner & n (\%) & $20(35,1)$ & $15(83,3)$ & $5(12,8)$ & $<0,001$ \\
\hline Déficit/ACV isquémico & n (\%) & $29(50,9)$ & $10(55,5)$ & $19(48,7)$ & 0,423 \\
\hline NIHSS 0-4 & n (\%) & $41(71,9)$ & $11(61,1)$ & $30(76,9)$ & 0,149 \\
\hline NIHSS 5-9 & n (\%) & $5(8,8)$ & $1(5,6)$ & $4(10,3)$ & \\
\hline NIHSS 10-19 & n (\%) & $9(15,8)$ & $4(22,4)$ & $5(12,8)$ & \\
\hline NIHSS 20 o más & n (\%) & $2(3,5)$ & $2(11,1)$ & $0 \quad(0)$ & \\
\hline Estenosis & $\mathrm{n}(\%)$ & $36(63,2)$ & $12(66,7)$ & $24(61,5)$ & 0,709 \\
\hline Oclusión & n (\%) & $14(24,6)$ & $6(33,3)$ & $8(20,5)$ & 0,296 \\
\hline Dilatación aneurismal & n (\%) & $6(10,5)$ & $2(11,1)$ & $4(10,3)$ & 1 \\
\hline Bilateral & n (\%) & $8(14,0)$ & $1(5,6)$ & $7(17,9)$ & 0,414 \\
\hline
\end{tabular}

NIHSS: National Institutes of Health Stroke Score. 
Figura 1. Localización de la disección por segmento arterial.

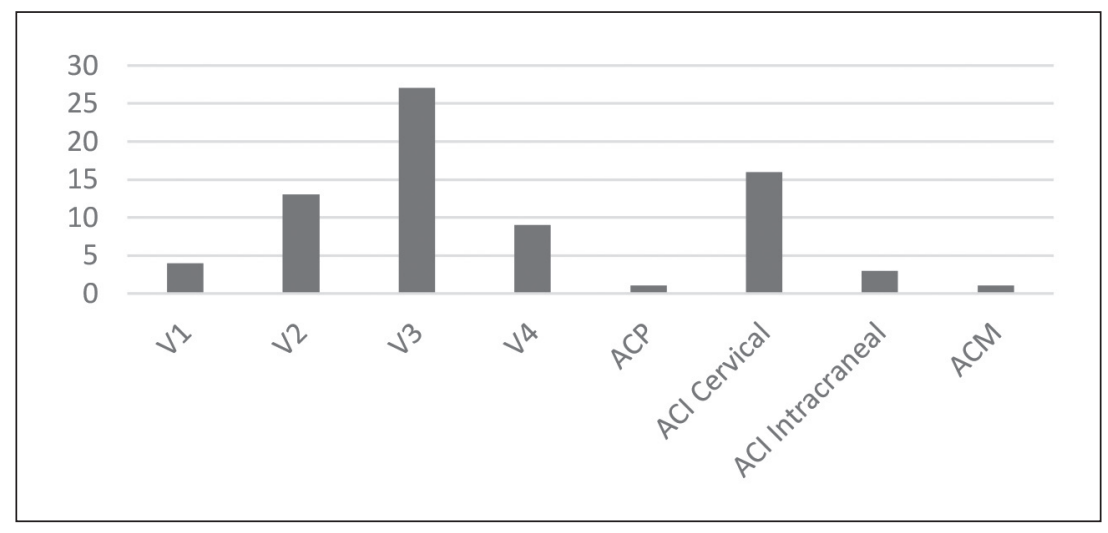

el segmento V3, estando comprometida en 27/39 pacientes lo que corresponde a $69,2 \%$, mientras que en circulación anterior, el segmento con mayor compromiso fue $\mathrm{C} 1$, al estar afectada en 16/18 pacientes, lo que corresponde a 88,8\% (Figura 1).

En agudo, 5 pacientes se sometieron a trombolisis endovenosa y 2 a trombectomía mecánica, en todos los casos en relación a embolía distal al sitio disecado.

En 2 pacientes se requirió la realización de una cranectomía descompresiva, en 1 caso por ACV extenso en territorio de arteria cerebral media y en el otro por una transformación hemorrágica en un infarto de arteria cerebelosa posteroinferior.

La terapia endovascular con stent se realizó en 13 casos (22,8\%), de ellos, en 5 el motivo fue compromiso bilateral con escasa colateralidad, en 3 progresión de la estenosis, en 2 la repercusión hemodinámica con hipoperfusión significativa, en 1 ACV recurrente, en 1 estenosis crítica inicial y en 1 dilatación aneurismática con manifestación clínica.

En relación a la terapia farmacológica, en 28/57 pacientes se indicó doble antiagregación plaquetaria con aspirina y clopidogrel, existiendo 3 argumentaciones al respecto: la presentación como un ACV isquémico menor o crisis isquémica transitoria, la instalación de un stent o la aparición de lesiones isquémicas en distinta temporalidad con patrón de embolía arterio-arterial (Tabla 3). La anticoagulación se utilizó en 7 pacientes, en 2 de ellos acenocumarol y en 5 un anticoagulante de acción directa, donde la indicación en la mayoría de los casos fue por patrón de embolía arterio-arterial.
Tabla 3. Tratamiento recibido

\begin{tabular}{|lc|}
\hline & n de pacientes \\
\hline Antigregación simple & 22 \\
\hline Antiagregación doble & 28 \\
\hline Anticoagulación & 7 \\
\hline Trombolisis & 5 \\
\hline Trombectomía & 2 \\
Stent & 13 \\
\hline Cranectomía & 2 \\
\hline
\end{tabular}

En la evaluación funcional a 3 meses, 52,6\% resultó estar en Rankin 0; 17,5\% en Rankin 1; $15,8 \%$ en Rankin $2 ; 1,8 \%$ en Rankin 3; $1,8 \%$ en Rankin 4; 3,5\% en Rankin 5 y 7\% en Rankin 6 (Figuras 2 y 3 ).

Un Rankin 0-2 se logró en 72,2\% de los pacientes con disección de circulación anterior, mientras que ese rango de funcionalidad se logró en 94,8\% de los pacientes con disección de circulación pos-

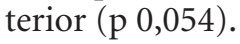

La recurrencia precoz de ACV o aparición de nuevas lesiones isquémicas a 3 meses fue de 5,2\%.

\section{Discusión}

Las características de los pacientes de nuestra serie es similar a lo reportado en la literatura, con un promedio de edad de 40 años y antecedente de trauma menor cercano a $20 \%$, sin embargo, 

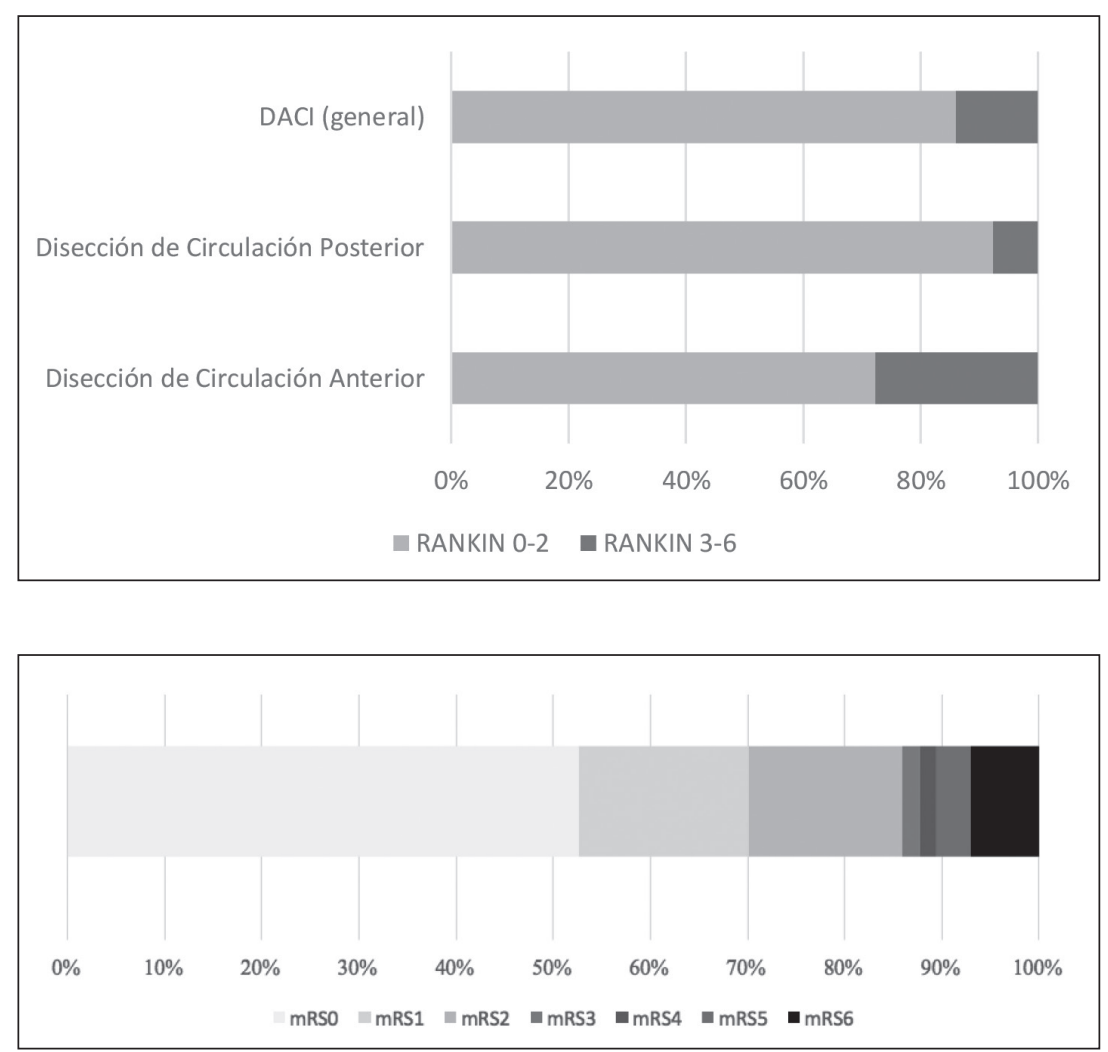

Figura 2. Funcionalidad a los 3 meses, medido a través de la escala de Rankin modificada.
Figura 3. Funcionalidad a los 3 meses de DACl en general, medido a través de la escala de Rankin modificada, en porcentajes. destaca la mayor proporción de mujeres, en particular en disecciones de arterias vertebrales, lo que probablemente se corresponde con una mayor prevalencia de patología menor del tejido conectivo en el género femenino ${ }^{7}$.

El dolor, ya sea como cefalea o cervicalgia, fue el síntoma más frecuente como forma de manifestación inicial, asociado en $35 \%$ con un síndrome de Horner, lo cual fue significativamente más frecuencia en relación a una afectación post ganglionar secundaria en una disección carotidea que secundaria a un infarto bulbar lateral por disecciones vertebrales, lo que es similar a lo reportado en el estudio CADISP ${ }^{8}$.

Es destacable la alta proporción de disecciones de circulación posterior en comparación a las de circulación anterior con una relación de 2:1, predominando el segmento V3 debido, probablemente, a la mayor movilidad y vulnerabilidad de las arterias extracraneales al estrés torsional, lo que también es válido para el segmento $\mathrm{C} 1$ en circulación anterior? ${ }^{9}$.
Con la mayor disponibilidad de estudios vasculares no invasivos para pacientes con cefalea o cervicalgia con banderas rojas, el diagnóstico de DACI es cada vez más frecuente en pacientes sin signología deficitaria o ACV, llegando a 50\% de los casos en nuestra serie, lo que es mayor a lo descrito en series de más larga data ${ }^{10-12}$. Además, en el grupo que sí presentó un ACV isquémico al momento del ingreso, la gravedad en la gran mayoría fue baja, predominando el ACV isquémico menor, y eso explica el alto porcentaje de pacientes en los que el equipo médico optó por una doble antiagregación plaquetaria por 3 semanas ${ }^{13}$. Es probable que en el futuro inmediato la tendencia sea a aumentar esta proporción o utilizar anticoagulación, pues existe evidencia creciente a favor de seleccionar precozmente a pacientes con mayor riesgo de evento cerebrovascular a través de la evaluación de reserva hemodinámica o la detección de señales microembólicas ${ }^{14}$, y migrar de la monoantiagregación a la antiagregación dual como en el $28 \%$ de la rama que recibió antiplaquetarios en el estudio CADISS ${ }^{4}$ 
o hacia la anticoagulación como en el grupo que obtuvo mejor outcome en TREAT-CAD ${ }^{15}$.

Cabe destacar el predominio del patrón estenooclusivo secundario a hematoma subintimal por sobre el pseudoaneurismático asociado a hematoma subadventicial, lo que, asociado a una evolución progresiva, condujo al equipo en un número importante de casos a decidir la realización de terapia endovascular con stent en particular por compromiso hemodinámico, demostrado por curvas intensamente postestenóticas en Doppler transcraneal, hipoperfusión en estudio imagenológico multimodal o pobre colateralidad en estudio angiográfico.

Si bien no hay diferencias significativas en la gravedad de la signología deficitaria ni en las características imagenológicas al momento del ingreso entre DACA y DACP, si hay una tendencia a una mejor funcionalidad en el grupo de disecciones de circulación posterior medido por ERm a 3 meses, lo que también debiese ser una variable a considerar en los siguientes ensayos clínicos, pues una diferencia mayor a 10\% en la proporción de disecciones vertebrales en las características basales de una de las rama de tratamiento podría ser un factor confundente al momento de comparar la eficacia de las intervenciones terapéuticas ${ }^{15}$.

El manejo terapéutico activo y dinámico que implica un seguimiento estrecho durante los primeros 7-14 días desde el inicio de los síntomas de esta patología alta, pero precozmente evolutiva ${ }^{15,16}$, explica que 9 de cada 10 pacientes que ingresaron al servicio con diagnóstico de DACI, a los 3 meses hayan logrado un nivel de funcionalidad que les permite la independencia en las actividades básicas de la vida diaria.

Agradecimientos: Agradecemos a Claudio Olmos G., Coordinador de la Unidad de Investigación Clínica INDISA, y a Marianela Honores C. de Servicios de Tecnología de la Información de Clínica INDISA por su contribución en la generación de la base de datos, con los estándares de anonimización aprobados por el CEC, para su posterior procesamiento estadístico.

\section{Referencias}

1. Shah Q, Messe SR. Cervicocranial arterial dissection. Curr Treat Options Neurol. 2007; 9 (1): 55-62.

2. Debette S, Leys D. Cervical-artery dissections: predis- posing factors, diagnosis, and outcome. The Lancet Neurology. 2009; 8 (7): 668-78.

3. von Babo M, De Marchis GM, Sarikaya H, Stapf C, Buffon F, Fischer U, et al. Differences and similarities between spontaneous dissections of the internal carotid artery and the vertebral artery. Stroke. 2013; 44 (6): 1537-42.

4. Markus HS, Levi C, King A, Madigan J, Norris J. Antiplatelet Therapy vs Anticoagulation Therapy in Cervical Artery Dissection: The Cervical Artery Dissection in Stroke Study (CADISS) Randomized Clinical Trial Final Results. JAMA Neurol 2019; 76: 657.

5. Brown SC, Falcone GJ, Hebert RM, Yaghi S, Mac Grory B, Stretz C. Stenting for Acute Carotid Artery Dissection. Stroke 2020; 51 (1): e3-e6. doi:10.1161/STROKEAHA.119.027827

6. Touze E, Gauvrit JY, Moulin T, Meder JF, Bracard S, Mas JL. Risk of stroke and recurrent dissection after a cervical artery dissection: A multicenter study. Neurology 2003; 61: 1347-51.

7. Castori M, Dordoni C, Valiante M, Sperduti I, Ritelli M, Morlino S, et al. Nosology and inheritance pattern(s) of joint hypermobility syndrome and Ehlers-Danlos syndrome, hypermobility type: A study of intrafamilial and interfamilial variability in 23 Italian pedigrees. Am J Med Genet A 2014; 164 (12): 3010-20.

8. Debette S, Grond-Ginsbach C, Bodenant M, Kloss M, Engelter S, Metso T, et al. Differential features of carotid and vertebral artery dissections: the CADISP study. Neurology. 2011; 77 (12): 1174-81.

9. Gupta S, Kumar VS. Cervical Artery Dissection-Related Stroke: Vascular Risk Factors May Have a Role. Ann Indian Acad Neurol. 2020; 23 (1): 129-30.

10. Engelter ST, Dallongeville J, Kloss M, Metso T, Leys $\mathrm{D}$, Brandt $\mathrm{T}$, et al. Thrombolysis in cervical artery dissection-data from the Cervical Artery Dissection and Ischaemic Stroke Patients (CADISP) database. Eur J Neurol. 2012; 19 (9): 1199-206.

11. Kloss M, Grond-Ginsbach C, Ringleb P, Hausser I, Hacke W, Brandt T. Recurrence of cervical artery dissection An underestimated risk. Neurology 2018; 90 (16): 13728.

12. Compter A, Schilling S, Vaineau C, Goeggel B, Metso $\mathrm{T}$, Southerland A, et al. Determinants and outcome of multiple and early recurrent cervical artery dissections. Neurology 2018; 91 (8): 769-80.

13. Wang Y, Wang Y, Zhao X, Liping L, Wang D, Wang C et al. Clopidogrel with aspirin in acute minor stroke or transient ischemic attack. N Engl J Med 2013; 369 (1): 11-9.

14. Brunser AM, Lavados PM, Cavada G, Muñoz-Venturelli 
P, Olavarría VV, Navia V, at al. Transcranial Doppler as a Predictor of Ischemic Events in Vertebral Artery Dissection. J Neuroimaging 2020; 30 (6): 890-5.

15. Engelter ST, Traenka C, Gensicke H, Schaedelin SA, Luft AR, Simonetti BG, et al; TREAT-CAD investigators. Aspirin versus anticoagulation in cervical artery dissection (TREAT-CAD): an open-label, randomised, non-infe- riority trial. Lancet Neurol 2021; S1474-4422(21)000442.

16. Morris NA, Merkler AE, Gialdini G, Kamel H. Timing of Incident Stroke Risk After Cervical Artery Dissection Presenting Without Ischemia [published correction appears in Stroke. 2018; 49 (10): e308]. Stroke 2017; 48 (3): 551-5.

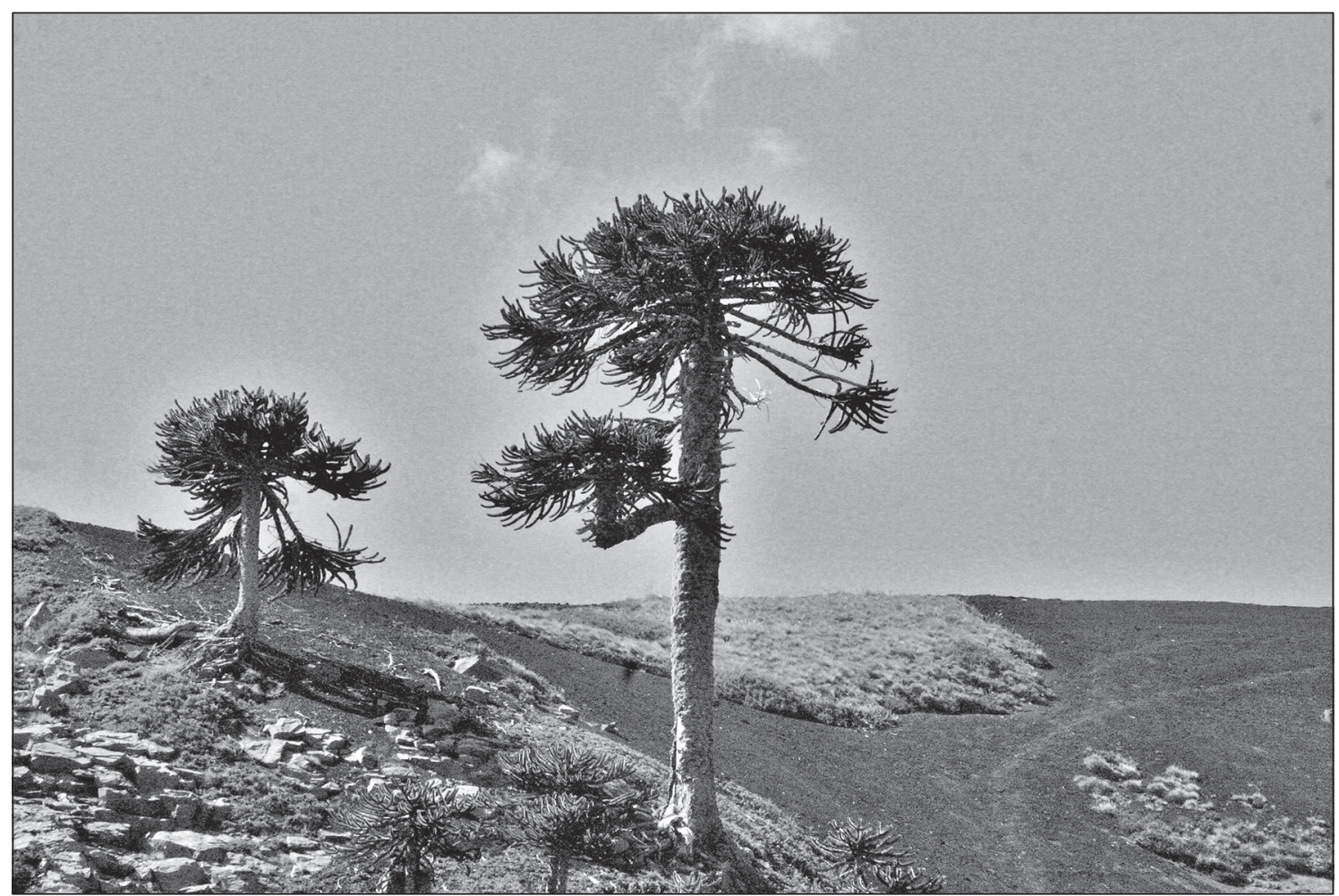

Araucarias, faldeo volcán Sollipulli, Melipeuco, Cautín Araucania. Dr. Jorge Sapunar Zenteno 